\title{
Explicit construction of an Autonomous Hamiltonian system exhibiting continual directed flow
}

\author{
A.D. Burbanks*, \\ D. Hennig, \\ C. Mulhern, \\ A.H. Osbaldestin \\ Department of Mathematics, University of Portsmouth, Portsmouth, PO1 3HF, UK
}

(Dated: June 18, 2012)

\begin{abstract}
We construct a prototypical example of a spatially-open autonomous Hamiltonian system in which localised, but otherwise unbiased, ensembles of initial conditions break spatio-temporal symmetries in the subsequent ensemble dynamics, despite time reversal symmetry of the equations of motion. Together with transient chaos, this provides the mechanism for the occurrence of a current. Transporting trajectories pass through transient chaos and subsequently cross surfaces of no-return, after which they perform solely regular motion so that the current is of continual ballistic nature.
\end{abstract}

PACS numbers: 05.60.Cd, 05.45.Ac, 05.60.-k, 05.45.Pq

\section{INTRODUCTION}

Transport of particles evolving in spatially periodic potentials has attracted much interest [1] (for a recent review, see [2]). In many studies, an external timedependent stochastic field with zero mean or a deterministic periodic forcing provide a current. Experiments on cold atoms in optical potentials have fuelled intensive studies of the Hamiltonian case [3]. By considering the symmetry of the external field and the underlying potential, the necessary conditions for rectification of the current have been enumerated [4], [5]: all symmetries that, to each trajectory, generate a counterpart moving in the opposite direction, need to be broken. A time-dependent external force that is periodic but not symmetric under time reversal [4]-[8] provides one means to achieve this. The so-called harmonic mixing mechanism can also be utilised to generate a non-vanishing current [9],[10]. Furthermore, coexisting regular and chaotic dynamics [6] are required, that is, one needs a mixed phase space. In extended chaotic systems a net current can be obtained as the time-averaged velocity of an ensemble of trajectories in the chaotic component of phase space, and the chaotic transport is ballistic and directed [6], [8].

Extensions to studies of autonomous Hamiltonian systems of one-dimensional billiard chains have followed [11], [12]. The requirement of chaotic motion necessitates at least two degrees of freedom. For example, [11] considers a classical magnetic billiard for particles carrying an electric charge, in which time-reversal invariance is broken by imposing an external static magnetic field perpendicular to the plane of motion. In order to achieve directed transport, the remaining spatial symmetry must also be broken, for example by placing asymmetric obstacles inside the billiard [11], [12]. Uni-directional motion in a serpent

*andrew.burbanks@port.ac.uk, Corresponding Author billiard chain has been reported in [13]. The requirement of a mixed phase space in a driven Hamiltonian system allows for the existence of chaotic trajectories that stick close to the boundaries of regular (transporting) regions, resulting in long periods of nearly regular motion [4]-[8]. As we discuss in [14]-[19], while sustained transport is achieved in the above scenario, the mechanism results in periods of regular motion that are intermittent in nature; their durations exhibit power-law decay statistics that seem to impede the overall transport. It may therefore be advantageous, when dealing with non-integrable systems, if chaos is of transient nature, serving to break separatrices and subsequently guide trajectories onto regular transporting motion.

The aim of recent work [14]-[19] has been to construct scenarios in autonomous Hamiltonian systems without mixed phase space, in which a directed flow arises even without the application of a time reversibility symmetry breaking external field. Specifically, we have shown that while such systems may as a whole be time-reversible, the practical necessity of considering (physically-relevant) sets of initial conditions that are localised with respect to a spatially-open component of the system, and furthermore are unbiased with respect to momentum in that degree of freedom, may nevertheless lead to a net current. In [14], a system was constructed from two integrable subsystems, together with a coupling term that vanishes asymptotically. Sets of initial conditions that are unbiased with respect to one subsystem, and are of practical interest due to their localisation in a spatially unbounded degree of freedom, can lead to a lack of symmetry in the ensuing ensemble dynamics. In addition, transient chaos serves to break separatrices and allows trajectories to escape from potential wells and settle asymptotically onto regular dynamics. This finds application [19] in accomplishing directed net motion in infinite lattice systems. Specifically, in the Holstein model [20], the charge being initially trapped in a confined region of the molecular chain constitutes localised initial conditions. Charge mo- 
tion along the chain can then be directed by taking into account non-reversion symmetric oscillators representing the intra-molecular vibrational degrees of freedom.

Here, we aim to provide a prototypical example of such symmetry violation in which chaos is again transient in nature, with the crucial difference that a region of solely regular transporting motion is reached by transporting trajectories in a finite time, once they leave a well-defined finite region of the phase space, rather than asymptotically. The system is again constructed from two independently integrable subsystems, however here the interaction term vanishes smoothly outside a finite "cutoff" radius. Transporting trajectories escape from the bottom of a potential well in the centre of the interaction region by undergoing transient chaos, which serves to break separatrices and guide the trajectory into the range of regular motion. The definition of the system allows the straightforward construction of explicit surfaces of noreturn outside the finite interaction region, which transporting trajectories pass through after a finite time. Once crossed, these surfaces cannot be re-crossed under the subsequent (independent) motions of the now-uncoupled integrable subsystems, which has the drastic implication that the directed net motion is provided solely by regular dynamics and is of continual ballistic nature.

\section{THE SYSTEM}

We consider a two degrees of freedom Hamiltonian,

$$
H(q, p, Q, P)=U(q)+V(Q)+W(q, Q)+\frac{1}{2}\left(p^{2}+P^{2}\right),
$$

modelling the conservative and deterministic dynamics of a particle whose coordinate $q$ evolves in a onedimensional periodic, spatially-symmetric washboard potential of unit period given by

$$
U(q)=U(q+1)=\frac{1}{2 \pi}(1-\cos 2 \pi q) .
$$

The particle interacts with a local oscillator of amplitude $Q$ evolving in an anharmonic potential,

$$
V(-Q) \neq V(Q)=e^{-Q}+Q-1
$$

The coupling between the particle and the anharmonic oscillator is provided by the interaction potential

$$
W(q, Q)=\left\{\begin{array}{l}
D\left(1-e^{\left(1-\left(1-x^{2}\right)^{-2}\right)}\right), \text { if }|x|<1, \\
D, \text { otherwise. }
\end{array}\right.
$$

with $x=(q-Q) / r$ for a fixed "cutoff radius", $r>0$. (The interaction potential, $W(q, Q)$, is illustrated in Fig. 1.) Note that the interaction depends on the distance $|q-Q|$. The parameter $D$ regulates the strength of the coupling between the particle and the anharmonic oscillator. The interaction function is smooth $\left(C^{\infty}\right)$ and can give rise to a

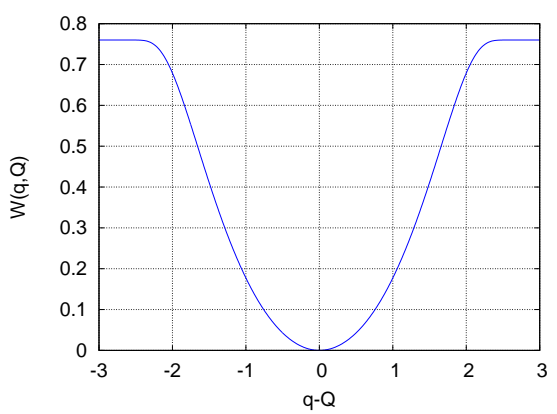

FIG. 1: The interaction term, $W(q, Q)$, plotted against the difference $q-Q$, for coupling parameter $D=0.76$ and cutoff radius $r=3$.

non-zero force only in the finite interval $x \in(-r, r)$. Thus there is a finite interaction region $|q-Q|<r$ within which the particle in the washboard potential and additional oscillator can exchange energy.

A broad class of interaction terms may be used to achieve similar results to those presented here. (In [14], an interaction term was used for which the interaction strength vanishes asymptotically.)

The equations of motion for the system are

$$
\begin{aligned}
& \ddot{q}=-\sin (2 \pi q)-\frac{\partial W}{\partial q}, \\
& \ddot{Q}=e^{-Q}-1-\frac{\partial W}{\partial Q}, .
\end{aligned}
$$

in which $-\frac{\partial W}{\partial Q}=\frac{\partial W}{\partial q}$, given by

$$
\frac{\partial W}{\partial q}= \begin{cases}\frac{4 D}{r} \frac{x \exp \left(1-\frac{1}{\left(1-x^{2}\right)^{2}}\right)}{\left(1-x^{2}\right)^{3}} & \text { if }|x|<1 \\ 0 & \text { otherwise }\end{cases}
$$

For $D=0$ the system decouples into two integrable subsystems and the dynamics is characterised by individual regular motions of the particle in the washboard potential, and bounded oscillations of the anharmonic degree of freedom, respectively. For $D \neq 0$, the subsystems interact in the region $|q-Q|<r$, exchanging energy. While the $Q$-oscillator performs solely bounded motion, there is the possibility that, for an escaping particle, the corresponding coordinate, $|q|$ (which, on energy shells of sufficiently high energy, represents the spatially-open component), attains a large enough value such that the particle leaves the interaction region and thus the related interaction forces, $\partial W / \partial q$ and $\partial W / \partial Q$, vanish, allowing for transient chaos [21]-[28].

\section{ESCAPING TRAJECTORIES}

We consider particles initially at rest at the bottom of the central well of the washboard potential with $p(0)=$ $q(0)=0$ (representing localised initial conditions in the 


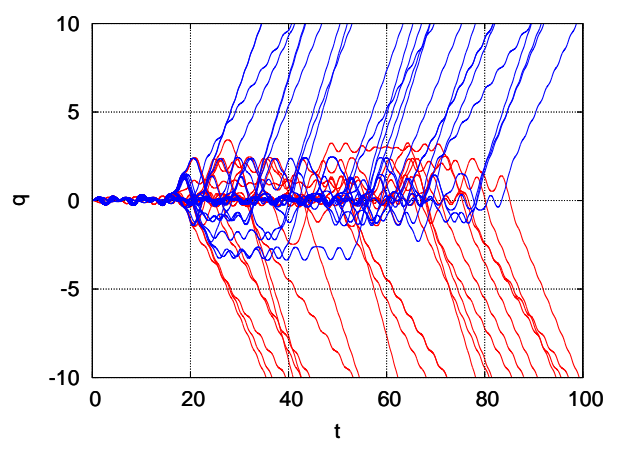

FIG. 2: A selection of transporting trajectories, showing the washboard coordinate, $q$, against time. After chaotic transients, the trajectories pass through surfaces-of-no-return and thereafter undergo regular motion. (On colour copy: trajectories settling on transport in the +ve (resp. -ve) $q$ direction are shown in blue (resp. red).)

spatially-open component, situated in the centre of the interaction region) and the total energy contained in the anharmonic oscillator. The particle can escape from the well only if it gains sufficient energy from its interaction with the excited oscillator, which acts as an energy deposit from which the particle can be fed. The total energy is fixed at $E=1.5$, which exceeds by several times the barrier height, $E_{b}=1 / \pi \simeq 0.318$, of the washboard.

We consider an iso-energetic ensemble of $10^{5}$ such initial conditions, uniformly distributed with respect to arclength on the level curve

$$
E=\frac{1}{2} P^{2}+V(Q)+W(0, Q)
$$

in the $(P, Q)$-plane (a topological circle). Note that the initial conditions are unbiased in the sense that they obey the symmetry $P \leftrightarrow-P$, and possess spatial symmetry with regard to the washboard potential.

Strikingly, for most initial conditions, the particle gains sufficient energy from the anharmonic degree of freedom that it escapes from the well and avoids becoming trapped in subsequent wells of the washboard potential. A selection of escaping trajectories is shown in Fig. 2.

A single escaping trajectory is shown in Fig. 3(a) which, after passage through a surface of no-return, moves directedly to the right (increasing coordinate, $q$ ) under regular rotational motion while the amplitude, $Q$, of the anharmonic degree of freedom, performs oscillations around the bottom of the potential $V(Q)$. After a chaotic transient the particle departs from the interaction region and, as we show in what follows, a point is reached at which both the interaction term in the equations of motion vanishes and at which subsequent independent (decoupled) motions of the particle and oscillator exclude the possibility of any future return to the interaction region, so that the observed behaviour is not merely a long-lived transient of the system; The dynamics of the two degrees

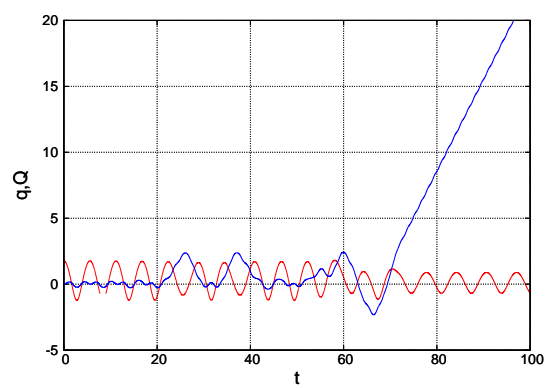

(a)

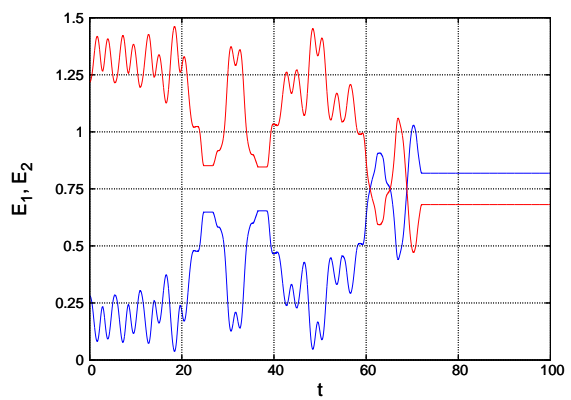

(b)

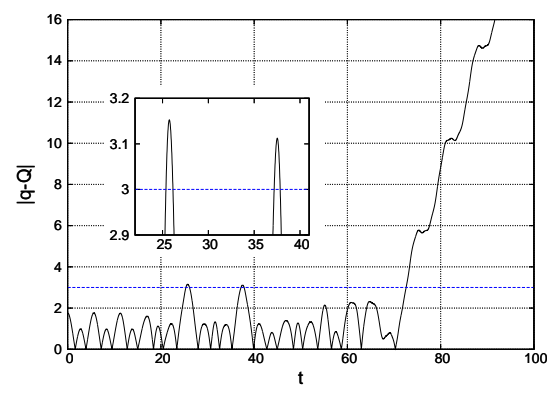

(c)

FIG. 3: Typical time evolutions (a) of the washboard coordinate, $q$, (blue on colour copy) and anharmonic oscillator coordinate, $Q$, (red on colour copy) plotted against time for a single escaping trajectory (here, $r=3, D=0.76$, and $E=1.5$ ). (b) Partial energies $E_{1}, E_{2}$, in the washboard and oscillator degree of freedom, respectively, (blue and red on the colour copy) plotted against time. (c) The distance $|q-Q|$, plotted against time. The cutoff radius, $r=3$, is indicated by the dotted horizontal line. The inset demonstrates two time intervals during which the trajectory momentarily leaves then re-enters the interaction region.

of freedom settles on individual regular motion and the Poincaré recurrence time is infinity.

It is instructive to associate partial energies with the particle and the oscillator. To this end, we define

$$
\begin{aligned}
& E_{1}=U(q)+\frac{1}{2} p^{2}+\frac{1}{2} W(q, Q), \\
& E_{2}=V(Q)+\frac{1}{2} P^{2}+\frac{1}{2} W(q, Q),
\end{aligned}
$$

in which we consider the interaction energy, $W$, to be shared equally between the particle and oscillator. Figure 3(b) illustrates the evolution of the partial energies for the trajectory shown in Fig. 3(a). Time intervals in which the particle leaves the interaction region momen- 

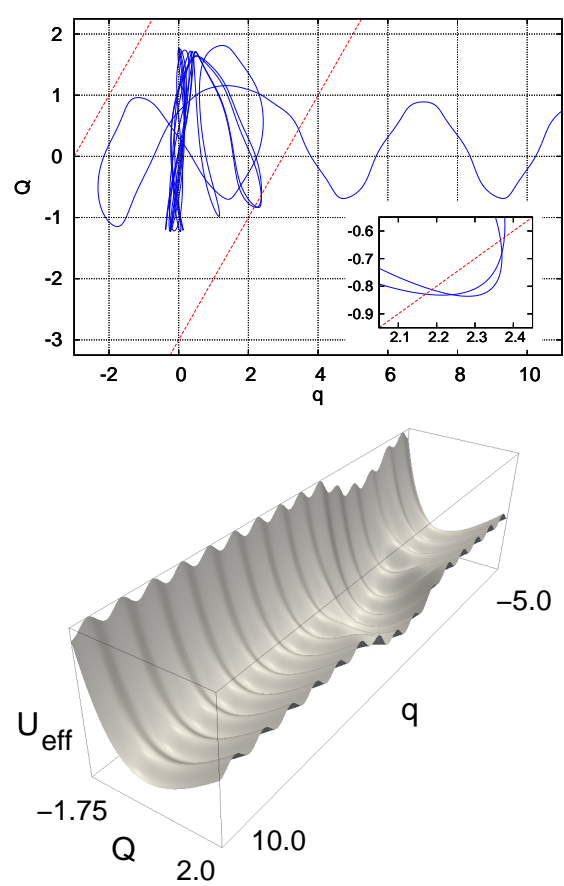

(a)

FIG. 4: (a) The transporting trajectory shown in Fig. 3(a), projected into the configuration space, $(q, Q)$. The boundary of the interaction region is indicated by the dotted lines (red on colour copy). The inset shows portions of the trajectory that momentarily leave then re-enter the interaction region. (b) The effective potential $U_{\text {eff }}(q, Q)$ of the system.

tarily are apparent (at $t \simeq 25$ and $t \simeq 37$ ), during which the partial energies remain constant. Ultimately, the particle crosses a surface of no-return in the phase space, beyond which no further interaction is possible $(t \gtrsim 70)$.

Figure 3(c) shows the time evolution of the distance, $|q-Q|$ in comparison with the interaction cutoff radius, $r$. In the inset, short time intervals during which the particle strays outside of the interaction region, prior to eventual escape, are readily apparent (cf. Fig. 3(b)). It is important to note that the small abrupt decreases (and subsequent increases) in the distance $|q-Q|$, observed in Fig. 3(c) at $t \simeq 76,82$, and 88 (when $|q-Q| \simeq 5.6,10.2$, and 14.6, respectively), are due to the independent motions of the washboard particle and (asymmetric) oscillator, associated with small time intervals during which $\partial Q / \partial t \simeq \partial q / \partial t$, rather than to interaction (those regions of the graph are not identical because the period of the anharmonic oscillator differs in general from that of the uncoupled motion in the washboard degree of freedom). This illustrates the importance of establishing the existence of surfaces of no-return for the dynamics, beyond which such independent motions cannot result in $|q-Q|<r$ at some later time. Figure 4(a) shows the trajectory projected into the configuration space $(q, Q)$, in comparison to the interaction region $|q-Q|<r$.

\section{ASYMMETRY AND PARTICLE CURRENT}

It is illustrative to consider the symmetry properties underlying the equations of motion, Eqs. (5) and (6), determined by the potential functions given in Eqs. (2)(4), which together give rise to an effective potential $U_{\text {eff }}(q, Q)=U(q)+V(Q)+W(q, Q)$. Figure 4(b) illustrates the (asymmetrical) effective potential. The washboard potential is periodic in $q$ with period 1 and invariant under reflection in $q$, i.e., $U(q)=U(-q)$, which amounts to symmetry with respect to every $q_{n}=n / 2$ with integer $n$. The interaction potential is invariant with respect to changes of the sign of its argument, viz., $(q-Q) \leftrightarrow-(q-Q)$. In contrast, the tilted anharmonic oscillator potential $V(Q)$ is without reflection symmetry and, consequently, the system of Eqs. (5) and (6) is not invariant under inversions $Q \leftrightarrow-Q$. As a result, no pairs of counter-propagating trajectories are produced by the localised, but otherwise unbiased, set of initial conditions chosen. In summary, although the equations of motion are time-reversible symmetric, the ensemble behaviour for the localised set of initial conditions defined in the previous section does not display this symmetry. This allows for the occurrence of a current.

Such net particle transport may be assessed quantitatively by the mean velocity; we define the current by the time average of the ensemble-averaged velocities, i.e.

$$
J_{D}=\frac{1}{T_{s}} \int_{0}^{T_{s}} d t^{\prime}\left\langle\dot{q}\left(t^{\prime}\right)\right\rangle,
$$

with simulation time $T_{s}$ where the average over the ensemble of $N=10^{5}$ particles defined earlier is given by

$$
\langle\dot{q}(t)\rangle=\frac{1}{N} \sum_{n=1}^{N} \dot{q}_{n}(t) .
$$

Note that the initial conditions are unbiased, obeying the symmetry $\dot{Q} \leftrightarrow-\dot{Q}$. The simulation time interval taken is $T=10^{5}$, being equivalent to almost $2 \times 10^{4}$ times the period of anharmonic oscillations near the bottom of a well of the washboard potential as well as of the potential of the anharmonic oscillator. The simulation time exceeds by far the time it takes for those particles which do escape to escape from the potential well. The dependence of the current on the coupling strength $D$ is displayed in Fig. 5. For under-critical values $D \lesssim 0.57$ the current is zero. For $0.57 \lesssim D \lesssim 0.78$ the current is mostly positive. There is a window of negative current around the coupling value $D \simeq 0.92$, then the current is again positive for $0.95 \lesssim D \lesssim 1.2$ The maximal current is attained for $D \simeq 1.05$. For coupling strength $D \gtrsim 1.2$ the current vanishes again.

The explanation for lack of current at high coupling is straightforward: outside the interaction region, i.e., for $|q-Q| \geq r$, the interaction potential is saturated at the value $W(q, Q)=D$. Recall that the energetic barrier height of the washboard potential is given by $1 / \pi=U(q+$ 


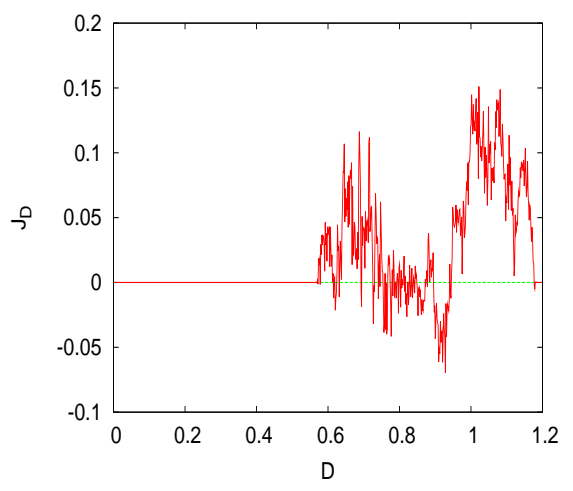

FIG. 5: The current as a function of the coupling strength $D$. The total energy of the system is $E=1.5$

$1 / 2$ ) with $q \in \mathbb{Z}$. Thus transport outside the interaction region is only possible if the total system energy obeys

$$
E>D+1 / \pi
$$

which implies that $D<E-1 / \pi$. At our chosen simulation energy, $E=1.5$, this implies that $D \lesssim 1.18169$.

As for the sharp transition between transporting and non-transporting motion at low coupling values, recall that (transient) chaos is required for the occurrence of a current. It suffices to apply the Toda criterion, which uses a local estimate of the rate of separation of neighbouring trajectories in phase space [29], [30], to provide an explanation in terms of borders of instability of motion in coordinate space. Specifically, the stability of the system is assessed by the time evolution of the corresponding linearised equations of motion, yielding a system for deviations from a given trajectory. The separation of trajectories grows exponentially if at least one of the eigenvalues of the corresponding stability matrix is real. Boundaries of such unstable regions correspond to changes in the sign of the Gaussian curvature of the effective potential surface $U_{\text {eff }}$. For our system the boundaries thus computed are in good agreement with the maximal extent of the non-transporting trajectories found at low coupling values (for brevity we omit the details; the particulars of such calculations are given in [14]).

\section{SURFACES OF NO-RETURN}

We now show that there exist trajectories that, once they have entered a certain region in phase space, provide continual transport of washboard particles.

Consider the Cauchy problem for the Hamiltonian in Eq. (1) with initial data $(p(0), q(0), P(0), Q(0))$. Let $E$ denote the total energy of the system, choosing $E>$ $D+1 / \pi$ and consider the energy shell given by $H=E$. We consider systems initially outside the interaction region, thus $|q(0)-Q(0)| \geq r$, the particle and oscillator are therefore decoupled, and the interaction potential is initially saturated; $W(q(0), Q(0))=D$. Further, let the particle degree of freedom contain sufficient energy that the particle is able to overcome the barrier height of the washboard potential; $U(q(0))+p(0)^{2} / 2=: E_{1}>1 / \pi$, which implies that the energy initially in the oscillator degree of freedom satisfies $V(Q(0))+P(0)^{2} / 2=: E_{2}<$ $E-D-1 / \pi$.

Now, let $Q_{\min }<0$, respectively $Q_{\max }>0$, denote the two solutions to the equation

$$
V(Q)=E-D-\frac{1}{\pi}>0
$$

Unless the system later enters the interaction region, the amplitude of the oscillator is thus bounded by $Q_{\min }<$ $Q<Q_{\max }$. In particular, initially $Q_{\min }<Q(0)<Q_{\max }$. To construct a surface that lies sufficiently far into the non-interaction region that independent motions of the particle and oscillator degrees of freedom cannot possibly lead the system back into the interaction region, it suffices to choose any $q(0)>Q_{\max }+r$, with $p(0)>0$ (or, alternatively, any $q(0)<Q_{\min }-r$, with $p(0)<0$ ). Thus the particle is on a transporting trajectory in the washboard degree of freedom travelling away from the oscillator, sufficiently far outside the interaction region that, even with the oscillator at its maximum amplitude, no interaction occurs. We claim that the two degrees of freedom remain decoupled and $q(t) \rightarrow+\infty$ monotonically; indeed, the region of phase space defined by

$$
\begin{aligned}
S=\left\{(q, p, Q, P) \in \mathbb{R}^{4}:\right. & H(q, p, Q, P)=E, \\
& q>Q_{\max }+r, p>0, \\
& \left.U(q)+p^{2} / 2>1 / \pi,\right\},
\end{aligned}
$$

(where $V\left(Q_{\max }\right)=E-D-1 / \pi$ and $\left.Q_{\max }>0\right)$ is forward invariant under the flow. (A surface of no return is given by replacing the above constraint on $q$ by $q=Q_{\max }+r$.) In order to prove the above, take any initial condition from the set $S$ and suppose (on the contrary) that the resulting trajectory re-enters the interaction region, i.e., there exists a time, $t_{2}>0$, for which $\left|q\left(t_{2}\right)-Q\left(t_{2}\right)\right|<r$. By continuity, there exists a first time, $t_{1}>0$ with $t_{1}<$ $t_{2}$, for which $\left|q\left(t_{1}\right)-Q\left(t_{1}\right)\right|=r$ and $|q(t)-Q(t)|>r$ for $0 \leq t<t_{1}$. Therefore the subsystems are decoupled during the closed time interval $0 \leq t \leq t_{1}$, with $E_{1}, E_{2}$ constant. We conclude that $Q_{\min } \leq Q\left(t_{1}\right) \leq Q_{\max }$ and, given $E_{1}>1 / \pi$ and $p(0)>0$, integrating the washboard subsystem gives $q\left(t_{1}\right)>q(0)>Q_{\max }+r \geq Q\left(t_{1}\right)+r$, hence $\left|q\left(t_{1}\right)-Q\left(t_{1}\right)\right|>r$, contradicting our assumption. In conclusion, as soon as the above region of phase space is reached (respectively, the region with $q<Q_{\min }-r$ and $p<0$, for which $q(t) \rightarrow-\infty$ as $t \rightarrow \infty$ ), no further redistribution of energy between the washboard degree of freedom and the anharmonic oscillator is possible. Most importantly, the washboard particle, being equipped with energy $E_{1}>1 / \pi$, moves in an unhindered and unidirectional manner, which establishes continual transport that cannot be terminated. 


\section{CONCLUSIONS}

We have provided an illustrative example of a system in which it is possible to obtain (continual) directed motion in autonomous Hamiltonian systems under the conditions that (i) transient chaos is supported and (ii) some of the degrees of freedom serve to break the time reversibility with regard to a set of unbiased localised initial conditions. The system is minimal in the sense that these two conditions constitute the only indispensable prerequisites. No additional modulation field is required in order to break the necessary spatio-temporal symmetries. The system that we present is constructed in such a way that, after passage through the transient chaos, transporting trajectories enter a region of phase space in which the system decouples fully into two separate integrable subsystems. Furthermore, those trajectories which reach the decoupled region may also pass through a surface of no-return, beyond which the subsequent (decoupled) motion cannot lead to re-entry of the coupled (interaction) region. Such trajectories undergo sustained regular motion, unbounded in the coordinates of the transporting degree of freedom.

This is in contrast to driven Hamiltonian systems with a mixed phase space, in which chaotic trajectories that spend a succession of episodes sticking close to regular transporting regions give rise to periods of regular motion whose durations exhibit power-law decay statistics.

With regard to extended chaotic systems, we note that a nonzero current can be obtained as the time-averaged velocity of an ensemble of trajectories in the chaotic component of phase space, and the chaotic transport proceeds ballistically and directedly. With our particular model system we have explored the possibility of inducing directed transport through transient chaos in an autonomous two degrees of freedom Hamiltonian system. That is, chaos is needed only in an initial stage of the dynamics to guide trajectories from the interior of separatrices onto sustained integrable rotational motion.
[1] H. Risken, The Fokker-Planck Equation (SpringerVerlag, New York, 1989).

[2] P. Hänggi and F. Marchesoni, Rev. Mod. Phys. 81, 387 (2009).

[3] T.S. Monteiro et al, Phys. Rev. Lett. 89, 194102 (2002); M. Schiavoni, L. Sanchez-Palencia, F. Renzoni, and G. Grynberg, Phys. Rev. Lett. 90, 094101 (2003); R. Gommers, S. Denisov, and F. Renzoni, Phys. Rev. Lett. 96, 240604 (2006).

[4] S. Flach, O. Yevtushenko, and Y. Zolotaryuk, Phys. Rev. Lett. 84, 2358 (2000).

[5] S. Denisov, S. Flach, A.A. Ovchinnikov, O. Yevtushenko, and Y. Zolotaryuk, Phys. Rev. E 66, 041104 (2002).

[6] H. Schanz, M.-F. Otto, R. Ketzmerick, and T. Dittrich, Phys. Rev. Lett. 87, 070601 (2001).

[7] S. Denisov and S. Flach, Phys. Rev. E 64, 056236 (2001); S. Denisov, J. Klafter, M. Urbakh, and S. Flach, Physica D 170, 131 (2002); S. Denisov, J. Klafter, and M. Urbakh, Phys. Rev. E 66, 046217 (2002).

[8] H. Schanz, T. Dittrich, and R. Ketzmerick, Phys. Rev. E 71, 026228 (2005)

[9] F. Marchesoni, Phys. Lett. A 119, 221 (1986).

[10] S. Savel'ev, F. Marchesoni, P. Hänggi, and F. Nori, Europhys. Lett. 67, 179 (2004).

[11] W. Acevedo and T. Dittrich, Prog. Theor. Phys. Suppl. 150, 313 (2003).

[12] H. Schanz and M. Prusty, Phys. A: Math. Gen. 38, 10085 (2005).

[13] M. Horvat and T. Prosen, J. Phys. A: Math. Gen. 37, 3133 (2004).

[14] D. Hennig, A.D. Burbanks, A.H. Osbaldestin, C. Mulhern, J. Phys. A: Math. Theor. 43345101 (2010).
[15] D. Hennig, A.D. Burbanks, C. Mulhern, A.H. Osbaldestin, Physical Review E 82, 026210 (2010).

[16] D. Hennig, A.D. Burbanks, A.H. Osbaldestin, J. Chem. Phys. 375 (2-3), 492-502

[17] D. Hennig, A.D. Burbanks, A.H. Osbaldestin, Physica D 238 (2009) 2273-2281.

[18] C. Mulhern, D. Hennig, A.D. Burbanks, A.H. Osbaldestin, Phys. Rev. E 83 (6), 066207 (2011).

[19] D. Hennig, A.D. Burbanks, A.H. Osbaldestin, Phys. Rev. E. 83 (3), 031121 (2011).

[20] T. Holstein, Ann. Phys. 8, 325 (1959).

[21] S. Bleher, C. Grebogi, and E. Ott, Physica D 46, 87 (1990).

[22] A. Pikovsky, J. Phys. A 25, L477 (1992).

[23] G. Contopolous, H.E. Kandrup, and D. Kaufman, Physica D 64, 310 (1993).

[24] T. Tel, J. Vollmer, and W. Breymann, Europhys. Lett. 35, 659 (1996).

[25] E. Ott Chaos in Dynamical Systems (Cambridge University Press, Cambridge, 2002).

[26] T. Tel, in Directions in Chaos (Ed.: Bai-lin Hao, World Scientific, Singapore, Vol. 3, 1990).

[27] T. Tel and M. Gruiz Chaotic Dynamics Cambridge University Press, Cambridge, 2006).

[28] M. Zaslavsky, Chaos in Dynamical Systems (Harwood, New York, 1985), Physics of Chaos in Hamiltonian Systems (Imperial College Press, London, 1998).

[29] M. Toda, Phys. Rev. Lett. A 48, 335 (1974).

[30] G. Benettin, R. Brambilla, and L. Galgani, Physica A 87, 381 (1977). 